\title{
Antibody Uptake Assay and In Vivo Imaging to Study Intracellular Trafficking of Notch and Delta in Drosophila
}

\author{
Lydie Couturier and François Schweisguth
}

\begin{abstract}
Notch signaling depends on regulated intracellular trafficking of the receptor and its ligands (Kopan and Ilagan, Cell 137:216-233, 2009; Le Borgne et al., Development 132:1751-1762, 2005). Here we describe two methods to study the intracellular trafficking of Notch and Delta in Drosophila. First, an ex vivo antibody uptake assay is used to monitor endocytosis of Notch and Delta by living cells in dissected explants (Le Borgne and Schweisguth, Dev Cell 5:139-148, 2003). Second, real-time imaging of fluorescent proteins that are expressed at physiological levels is used to study trafficking of Notch in living flies (Venken et al., Science 314:1747-1751, 2006; Couturier et al., Nat Cell Biol 14, 131-139, 2012).
\end{abstract}

Key words Live imaging, Antibody uptake, Endocytosis assay, Notum dissection, Drosophila

\section{Introduction}

Notch regulates binary fate decisions in various developmental contexts, including asymmetric cell divisions $[1,2]$. In this environment, generation of cell fate diversity and self-renewal of stem cells may rely on the unequal segregation of regulators of the intracellular trafficking of Notch and Delta. In Drosophila, each bristle sensory organ consists of four distinct cells that are generated via a series of stereotyped asymmetric divisions from a single Sensory Organ Precursor cell (SOP) [3]. At pupal stages (during metamorphosis), SOPs divide asymmetrically to generate an anterior pIIb cell and a posterior pIIa cell in the notum (a single-layered epithelium forming the dorsal thorax). The binary pIIa versus pIIb decision is regulated by Notch; inhibition of Notch specifies pIIb whereas activation of Notch leads to pIIa specification. Two cell fate determinants, Numb and Neuralized (Neur), localize at the anterior cortex of mitotic SOPs, are specifically inherited by pIIb cells and act in parallel to set up directional Notch signaling $[4,5]$. Numb has been proposed to inhibit the recycling of Notch indirectly via its interaction with Sanpodo (Spdo), a four-pass transmembrane 
protein that regulates Notch endocytosis [6-8]. The E3 ubiquitin ligase Neur regulates ubiquitin-dependent endocytosis and activity of the ligand Delta (Dl), thereby activating Notch in pIIa $[4,9]$.

To further elucidate the activity of Notch and its ligands during cell fate determination, we have developed two assays. The first (Subheading 3.1) is an antibody uptake assay which allows one to study endocytosis, i.e., internalization, of Notch and Dl in the pupal notum. Briefly, the single-layered epithelium corresponding to the pupal notum is dissected and cultured in presence of antibodies recognizing an extracellular epitope of $\mathrm{Dl}$ or Notch. Following medium changes and fixation, the uptake of anti-Dl or anti-Notch antibodies is revealed using secondary antibodies. Thus, this ex vivo assay monitors the internalization of Notch and its ligand $\mathrm{Dl}[4,6]$ and can be used to genetically study the regulation of Notch and Dl endocytosis [10-12]. The second (Subheading 3.2) allows one to study the intracellular trafficking of Notch in living flies, using GFP-tagged versions of Notch and its regulators Numb and Spdo that we have generated $[6,7,13]$. Here, we describe how to study Notch intracellular trafficking by live imaging in Drosophila with these flies.

\section{Materials}

\subsection{Materials for Method A: Antibody Uptake Assay to Monitor the Trafficking of Notch and Its Ligand Delta in Drosophila}

1. Dissection Petri dishes layered with Rhodorsil RTV-2 silicone (or similar cured silicone elastomer such as Sylgard ${ }^{\circledR}$ ).

2. Dissection tools: Dumont \#5 forceps, Vannas micro-scissors (75 $\mathrm{mm}$ straight), fine Minutien pins $(0.15 \mathrm{~mm}$ diameter).

3. Binocular stereomicroscope and confocal microscope.

4. Small glass incubation dishes.

5. Schneider's Drosophila medium containing $1 \%$ fetal calf serum.

6. PBS $10 \times$ to prepare PBT (PBS $1 \times$ with $0.1 \%$ Triton X100).

7. $4 \%$ paraformaldehyde (PFA) in PBS $1 \times(1 \mathrm{ml}$ aliquots kept at $-20{ }^{\circ} \mathrm{C}$; aliquots prepared from PFA powder).

8. Primary antibodies: mouse monoclonal anti-Delta ${ }^{\mathrm{ECD}}$ (C594.9B) directed against the extracellular domain of $\mathrm{Dl}$ [14] and anti-Notch ${ }^{\mathrm{ECD}}(\mathrm{C} 458.2 \mathrm{H})$ directed against EGF repeats 12-20 of the extracellular domain of Notch [15]; concentrates obtained from the Developmental Studies Hybridoma Bank, University of Iowa.

9. Fluorescently conjugated anti-mouse secondary antibodies.

10. Prepare $50 \%$ glycerol $(\mathrm{v} / \mathrm{v}), 50 \%(\mathrm{v} / \mathrm{v})$ PBS $\mathrm{l} \times$ solution.

11. Mounting medium: $20 \%(\mathrm{v} / \mathrm{v})$ PBS $1 \times, 80 \%(\mathrm{v} / \mathrm{v})$ glycerol, $2 \%$ (w/v) $N$-propyl-galate (Sigma). 
2.2 Materials for

Method B: In Vivo

Imaging of

Intracellular

Trafficking of Notch

Using a GFP-Tagged

Protein in Drosophila
1. Transgenic flies carrying $\mathrm{P}\{\mathrm{acman}\}$ Bacterial Artificial Chromosome (BAC) transgenes encoding GFP-tagged Notch (N-iGFP, Notch intracellular Green Fluorescent Protein), Numb (Numb-GFP), or Spdo (Spdo-iGFP). These transgenes were obtained by modifying BAC clones generated by Venken and colleagues [16]. They encode fully active proteins expressed at physiological levels, as demonstrated using a genomic rescue assay $[6,7]$ (see also Chapter 3).

2. Transgenic flies carrying RFP-tagged markers that are expressed specifically in SOPs, such as neur-PH-RFP (expressing the PIP2 binding domain of PLC $\gamma 1$ fused to mRFPl under the control of a neur SOP-specific enhancer) or neur-H2B-RFP (expressing Histone2B fused to mRFPl in SOPs) $[6,7]$.

3. Forceps (Dumont \#5), Vannas micro-scissors $(75 \mathrm{~mm}$ straight), fine Minutien pins (0.15 mm diameter).

4. Binocular stereomicroscope (for dissection); fluorescent microscope or confocal microscope (for imaging).

5. Small Petri dishes (diameter $55 \mathrm{~mm}$ ) and temperaturecontrolled fly incubator $\left(25^{\circ} \mathrm{C}\right)$.

6. Standard microscope slides.

7. Custom-made metallic spacers $(10 \times 10 \times 0.7 \mathrm{~mm})$; can be replaced by a stack of $4-5$ standard coverslips $(0.17 \mathrm{~mm})$ glued together using nail polish.

8. Double-sided tape.

9. Voltalef oil 10S (VWR \#24627-188); other high viscosity Halocarbon oil can also be used.

\section{Methods}

3.1 Antibody Uptake Assay to Monitor the Trafficking of Notch and Its Ligand Delta in Drosophila

\subsubsection{Selection} and Staging of Pupae

3.1.2 Dissection of Staged Pupae
All steps are performed at room temperature unless otherwise specified.

1. Select pupae of the appropriate genotype at the onset of metamorphosis, i.e., at $0 \mathrm{~h}$ After Puparium Formation ( $0 \mathrm{~h} \mathrm{APF})$ by visual inspection of the vial every $30 \mathrm{~min}$ (check for eversion of the anterior spiracles in immobile larvae). Soft white pre-pupae can be gently removed from the vial using a soft-bristled brush.

2. Place collected pre-pupae in a petri dish with a moistened tissue paper and place in an incubator: SOPs divide around 16.5 h APF at $25^{\circ} \mathrm{C}$, at $24 \mathrm{~h} \mathrm{APF}$ at $21^{\circ} \mathrm{C}$, and at $33 \mathrm{~h} \mathrm{APF}$ at $18{ }^{\circ} \mathrm{C}$.

1. Transfer staged pupae to the dissecting dish ventral side up.

2. Pin down pupae by inserting two fine needles through the abdomen (two needles are necessary to prevent rotation). 
3.1.3 Antibody Uptake Assay
3. Cover pupae with Schneider's Drosophila medium containing $1 \%$ fetal calf serum.

4. Using the micro-scissors, cut the anterior tip of the pupal case. Carefully insert one blade of the micro-scissor between the body of the immature fly and the pupal case and make a medial cut into the pupal case (along the ventral midline). Using forceps, tear and remove the pupal case. The body of the immature fly should then be free of the pupal case except over its abdomen [17].

5. Using the micro-scissors, cut through the pupal head at the level of the eyes to remove the anterior part (from now on, pieces of tissue floating in the pupal body cavity will escape).

6. Carefully insert one blade of the micro-scissor inside the head opening, make a cut laterally at the level of the developing wing down to the abdomen, and repeat on the contralateral side.

7. Lift the ventral part of the immature fly and detach it by making a transverse cut at its base, i.e., at the level of the abdomen.

8. Using a Pasteur pipette, gently flush once the inside of the pupa. Speed (hence practice) is the trick, so dissect and process pupae one at a time.

1. Remove the medium and replace it with fresh medium containing the anti-Delta ${ }^{\mathrm{ECD}}$ (or anti-Notch ${ }^{\mathrm{ECD}}$ ) antibody (1:10$1: 100)$ to perform the antibody uptake assay of the freshly dissected notum (see Note 1). $1 \mathrm{ml}$ of antibody solution is required to cover the dissected pupa in the dissecting dish. Alternatively, particularly if the antibody is precious, it is possible to wipe out the dissecting dish around the dissected pupa and to cover the pupa with a $0.1 \mathrm{ml}$ drop of antibody solution.

2. Place the dissecting dish in a humidified box and incubate at $25{ }^{\circ} \mathrm{C}$ for the desired period (5-30 min) (see Note 2). Internalization of $\mathrm{Dl}$ can be detected within $3 \mathrm{~min}$ and a strong signal can be measured within $10 \mathrm{~min}$ in SOPs. In our hands, the internalization of Notch is less efficient than Delta and longer incubation ( $15 \mathrm{~min}$ ) is required to detect the internalization of Notch.

3. Optional: the antibody-containing medium can be replaced by fresh medium for a defined period of chase (5-30 min) prior to fixation.

4. Remove the antibody-containing medium and wash once with Schneider's Drosophila medium.

5. Fix the pupa directly in the dissecting dish for 20 min under a chemical fume hood by adding $1 \mathrm{ml}$ of fixative. 
6. Wash $3 \times 5 \mathrm{~min}$ in PBT.

7. Complete the notum dissection by trimming down the notum on both sides (dorsal to the pupal wings) using micro-scissors to keep only the dorso-central part of the dorsal thorax (see Note 3). Cut the notum away from the abdomen and transfer it using forceps into a small glass incubation dish. To avoid damaging the notum, grasp the dissected tissue by the head part.

8. To evaluate variability in the uptake, repeat the internalization of anti-Dl ${ }^{\mathrm{ECD}}$ (or anti-N ${ }^{\mathrm{ECD}}$ ) with 5-10 nota. Pool these nota after the post-fixation dissection step and before proceeding to the antibody detection step below.

9. To detect internalized mouse monoclonal antibodies, incubate the notum for $60 \mathrm{~min}$ with fluorescently labeled antimouse secondary antibodies $(1: 2,000)$ in PBT on a rotating platform.

10. Optional: to compare the distribution of internalized antiDlECD with endogenous $\mathrm{Dl}$, primary guinea-pig anti-Dl antibodies (1:2,000; obtained from M. Muskavitch) [18] can be combined with the fluorescently labeled anti-mouse secondary antibodies. If so, proceed, and an additional secondary antibody incubation step will be required.

11. Optional: appropriate primary antibodies against sensory cell and/or endosome markers can also be combined with the fluorescently labeled anti-mouse secondary antibodies. If so, proceed, and an additional secondary antibody incubation step will be required.

12. Wash $3 \times 10 \mathrm{~min}$ in PBT.

13. Wash and equilibrate in $50 \%$ glycerol in PBS $1 \times$.

14. Using forceps, transfer the nota into a drop of mounting medium deposited on a standard microscope slide, cover with coverslip and seal.

15. Under the binocular scope, mount nota dorsal up (i.e., apical up for the notum epithelium).

16. Analyze the results by confocal microscopy.

3.2 In Vivo Imaging of Intracellular Trafficking of Notch Using a GFP-Tagged Protein in Drosophila

3.2.1 Mounting Staged Pupae for Live Imaging
1. Place a piece of double-sided tape onto the microscope slide. Transfer pupae (staged as in Subheading 3.1.1; now brown and hard) from the petri dish and adhere the pupal case to the double-sided tape with the ventral side down ( see Note 4).

2. Using forceps, grasp the anterior edge of the operculum (anterior-dorsal tip of the pupal case, just above the head of the immature fly), lift and remove. Carefully insert one blade of the micro-scissor between the body of the immature fly (at the level of the humerus) and the pupal case and make a cut into the pupal case. Tear apart the fragments of the pupal case 
3.2.2 Live Imaging

covering the dorsal thorax (notum) using the forceps and/or micro-scissor. Stick the removed pieces onto the double-sided tape. It is not necessary to remove the part of the pupal case that covers the abdomen. Be careful not to puncture the pupal body as it is soft and very fragile.

3. Place spacers on the double-sided tape on both sides of the pupae.

4. Using a Pasteur pipette, spread a small amount of Voltalef oil into a thin layer covering a coverslip (see Note 5). Deposit the coverslip onto the spacers, oiled face down. Check that the notum is in direct contact with the coverslip. Tape the coverslip onto the slides using regular tape. Pupae are now ready for imaging.

1. Perform live imaging at $20 \pm 2{ }^{\circ} \mathrm{C}$ using a microscope equipped with a $63 \times$ objective (HCX PL APO CS, N.A. 1.3). Inverted or upright microscopes may be used. Also, scanning or spinning disk confocal microscopes may be used. Using a PerkinElmer UltraVox Spinning Disk equipped with a CSU-XI disk on an inverted Zeiss microscope, 488 and 561 lasers and two Hamamatsu ImagEM EM-CCD cameras for simultaneous acquisition in the green and red channels (see Note 6), movies of dividing SOPs and or $\mathrm{pIIa} / \mathrm{pIIb}$ cell pairs can be obtained with high spatial and temporal resolution by acquiring a stack of 15-20 images with a $\Delta \mathrm{z}$ of $0.3 \mathrm{~mm}$ and an acquisition time of 100 ms every $3-5 \mathrm{~s}$. Under these conditions, time-lapse series of 100-150 stacks can be acquired with reduced bleaching. Imaging conditions need to be adapted to the intensity of fluorescence as well as the size and mobility speed of the objects that are tracked over time. Microscope control and image acquisition is driven under Volocity. Fiji/ImageJ is used for post-acquisition processing of the images.

2. Following imaging, the microscope slide with the imaged pupae can be transferred into a humidified chamber to let pupae develop into adult flies. If adult flies need to be recovered, remove the coverslip and double-sided tape around the pupae.

\section{Notes}

1. The antibody uptake assay can be adapted to monitor the internalization of anti-D $\mathrm{I}^{\mathrm{ECD}} / \mathrm{N}^{\mathrm{ECD}}$ antibodies in real time by forming in vitro complexes between anti- $\mathrm{Dl}^{\mathrm{ECD}} / \mathrm{N}^{\mathrm{ECD}}$ and fluorescently labeled anti-mouse $\mathrm{F}\left(\mathrm{ab}^{\prime}\right)_{2}$ fragments [19]. Additionally, the time-course of internalization can be studied by incubating the notum sequentially with two anti- $\mathrm{Dl}^{\mathrm{ECD}}$ / anti-mouse $\mathrm{F}\left(\mathrm{ab}^{\prime}\right)_{2}$ fragments solutions that differ only by the 
fluorochrome coupled to the $\mathrm{F}\left(\mathrm{ab}^{\prime}\right)_{2}$ fragments (our unpublished results).

2. A $4{ }^{\circ} \mathrm{C}$ incubation step is not required for cell surface binding of the antibody prior to its internalization. A $4{ }^{\circ} \mathrm{C}$ incubation step may actually promote the antibody-mediated clustering of epitopes, thereby promoting their internalization.

3. The antibody uptake assay can be applied to genetically mosaic flies carrying clones of mutant cells, thereby permitting a genetic analysis of the process of Notch and/or Dl internalization.

4. Using fully functional tagged proteins expressed at physiological levels is highly desirable, since trafficking routes may depend on the association/dissociation kinetics of components of the endocytic machinery and, therefore, on cargo concentration. Overexpression of fluorescently tagged proteins should therefore be avoided to study intracellular trafficking. Similarly, overexpression of endosomal markers, such as FP-tagged Rab GTPases, should be avoided as this may impact upon endosomal dynamics. Hence we recommend using genomic tagged $\mathrm{P}\{\mathrm{acman}\}$ BAC clones available at BACPAC Resources, Children's Hospital Oakland Research Institute (http://bacpac.chori.org/) [16].

5. It is important to minimize the amount of oil on the coverslip when using an upright microscope for live imaging. Indeed, too much oil will result in oil sliding along the side of the pupa.

It is also important to ensure that the notum is in direct contact with the coverslip to optimize the light path, but do not apply too much pressure on the pupa to increase the size of the contact region between the pupa and the coverslip (i.e., to maximize the field of view) as the living tissue may react to physical pressure.

The refraction index of the Voltalef oil is very similar to the refraction index of the oil used for the objective.

6. Using two EM-CCD cameras for fast simultaneous acquisition allows both channels to be acquired at the exact same time. This may be important to study the co-localization of red and green markers into rapidly moving endosomes. The spatial registration of the signals acquired by the two cameras should be verified prior to imaging by stopping the rotation of the spinning disk and imaging the two channels through the pinholes of the disk.

While a spinning disk is best suited for the imaging of rapidly moving structures, laser scanning microscopes can also be used under fast scanning modes. Excitation should be minimized to limit both phototoxicity and bleaching. 


\section{Acknowledgments}

This work was supported by the Fondation pour la Recherche Médicale (DEQ20100318284). The antibody uptake assay was initially developed together with $\mathrm{R}$. Le Borgne.

\section{References}

1. Kopan R, Ilagan MX (2009) The canonical Notch signaling pathway: unfolding the activation mechanism. Cell 137:216-233

2. Le Borgne R, Bardin A, Schweisguth F (2005) The roles of receptor and ligand endocytosis in regulating Notch signaling. Development 132:1751-1762

3. Gho M, Bellaiche Y, Schweisguth F (1999) Revisiting the Drosophila microchaete lineage: a novel intrinsically asymmetric cell division generates a glial cell. Development 126:3573-3584

4. Le Borgne R, Schweisguth F (2003) Unequal segregation of Neuralized biases Notch activation during asymmetric cell division. Dev Cell 5:139-148

5. Rhyu MS, Jan LY, Jan YN (1994) Asymmetric distribution of Numb protein during division of the sensory organ precursor cell confers distinct fates to daughter cells. Cell 76:477-491

6. Couturier L, Vodovar N, Schweisguth F (2012) Endocytosis by Numb breaks Notch symmetry at cytokinesis. Nat Cell Biol 14: 131-139

7. Couturier L, Mazouni K, Schweisguth F (2013) Numb localizes at endosomes and controls the endosomal sorting of notch after asymmetric division in Drosophila. Curr Biol 23:588-593

8. Cotton M, Benhra N, Le Borgne R (2013) Numb inhibits the recycling of Sanpodo in Drosophila sensory organ precursor. Curr Biol 23:581-587

9. Yamamoto S, Charng WL, Bellen HJ (2010) Endocytosis and intracellular trafficking of Notch and its ligands. Curr Top Dev Biol 92:165-200

10. Giagtzoglou N, Yamamoto $S$, Zitserman D et al (2012) dEHBPl controls exocytosis and recycling of Delta during asymmetric divisions. J Cell Biol 196:65-83
11. Daskalaki A, Shalaby NA, Kux K et al (2011) Distinct intracellular motifs of Delta mediate its ubiquitylation and activation by Mindbombl and Neuralized. J Cell Biol 195:1017-1031

12. Le Borgne R, Remaud S, Hamel S et al (2005) Two distinct E3 ubiquitin ligases have complementary functions in the regulation of Delta and Serrate signaling in Drosophila. PLoS Biol 3:e96

13. Venken KJ, He Y, Hoskins RA et al (2006) $\mathrm{P}[\mathrm{acman}]$ : a BAC transgenic platform for targeted insertion of large DNA fragments in D. melanogaster. Science 314:1747-1751

14. Qi H, Rand MD, Wu X et al (1999) Processing of the Notch ligand Delta by the metalloprotease Kuzbanian. Science 283:91-94

15. Diederich RJ, Matsuno K, Hing H et al (1994) Cytosolic interaction between deltex and Notch ankyrin repeats implicates deltex in the Notch signaling pathway. Development 120: 473-481

16. Venken KJ, Carlson JW, Schulze KL et al (2009) Versatile P[acman] BAC libraries for transgenesis studies in Drosophila melanogaster. Nat Methods 6:431-434

17. Jauffred B, Bellaiche Y (2012) Analyzing frizzled signaling using fixed and live imaging of the asymmetric cell division of the Drosophila sensory organ precursor cell. Methods Mol Biol 839:19-25

18. Klueg KM, Parody TR, Muskavitch MA (1998) Complex proteolytic processing acts on Delta, a transmembrane ligand for Notch, during Drosophila development. Mol Biol Cell 9:1709-1723

19. Coumailleau F, Furthauer M, Knoblich JA et al (2009) Directional Delta and Notch trafficking in Sara endosomes during asymmetric cell division. Nature 458:1051-1055 